\title{
Research on the Mutation Mechanism of Victim's Psychology and Behavior after Natural Disaster Based on Catastrophe Theory
}

Weiwei Zhang ${ }^{1}, X u a n h u a X u^{1}$, Bin Pan $^{2}$

${ }^{1}$ School of Business, Central South University, Changsha 410083, China

${ }^{2}$ Instituteof Financial Research, Wenzhou University, Wenzhou 325027, China

\section{基于突变理论的自然灾害灾民个体心理行为状态 突变机理研究 \\ 张威威 ${ }^{1}$, 徐选华 ${ }^{1}$, 潘森 ${ }^{2}$ \\ 1 中南大学商学院, 长沙 410083 , 中国 \\ 2 温州大学金融研究院, 温州 100875, 中国}

\begin{abstract}
Aiming at the problem of victim's rational and irrational behavior during recovery stage in natural disaster, this paper analyzed the victim's psychological and behavioral characteristics and influencing factors during recovery stage based on lots of literatures and an interview with victims. Besides, a cusp catastrophe model is constructed to explore the mutation mechanism of victim's psychology and behavior at the role of physical demand factors and emotional needs factors. Finally, the paper put forward some strategies to cope with victim's psychology and behavior during recovery stage based on the cusp catastrophe model analysis.
\end{abstract}

Keywords: Natural disaster; Victim's psychology and behavior; Cusp catastrophe model; Mutation mechanism; Coping strategy

摘要: 针对自然灾害恢复期灾民理性行为和非理 性行为之间的变化问题, 根据文献分析和灾区调 研分析了恢复期灾民心理行为的特征及影响因 素, 构建了灾民心理行为形成机制图和灾民心理 行为尖点突变模型, 探讨了恢复期灾民心理行为 在物质需求因素和情感需求因素作用下的突变 机理, 分析了两种需求因素的不同作用, 应用灾 区调研数据对模型进行了局部验证。最后基于模
型分析给出了应对灾民心理行为突变的两个策 略。

关键词: 自然灾害; 灾民心理行为; 尖点突变模 型; 突变机理; 应对策略

1 引言

随着全球气候变化问题的日益突出, 各类自 然灾害事件发生的频率不断增大, 影响范围越来 越广。据民政部统计, 我国 2014 年各类自然灾 害共造成 24353.3 万人次受灾, 1583 人死亡, 601.7 万紧急转移安置, 直接经济损失高达 3373.8 亿元 ${ }^{[1]}$ 。自然灾害不仅造成大量人员伤亡 和经济损失, 也给受灾群众的心理造成了不同程 度的创伤, 在短期甚至长期内都可能影响个体的 行为 ${ }^{[2]}$, 许多案例已经表明灾害情景下焦虑情绪 会促使受灾儿童做出一些攻击性行为 ${ }^{[2]}$, 这些负 面心理行为体验会增大救援和安置困难, 如果得 不到及时有效的缓解而被逐渐累积, 很容易外化 为违法行为, 甚至演化为群体性事件 ${ }^{[4]}$, 降低社 会的稳定性和安全性 ${ }^{[6]}$ 。灾民行为分析对于危机 管理、灾害应急响应、疏散计划实施有着重要作 用 ${ }^{[8]}$, 也是突发公共事件应急管理中的核心科学 问题之一 ${ }^{[9]}$ 。因此, 为有效救援和阻断个体事件 演化升级, 科学制定灾后救援策略, 需要深入分 析自然灾害发生后受灾群众的个体心理行为状 态突变机理。 
Risk Analysis and Crisis Response in Big Data Era (RAC-16)

许多研究者对气候变化、自然灾害发生后受 灾群众的心理及行为进行了相关研究。Doherty ${ }^{[10]}$ 分析了气候变化对人们心理的三种影响: 直接心 理影响 (急性应激障碍或创伤后应激障碍); 间 接心理影响 (幸福感下降); 社会心理影响 (社 会冲突)。Norris ${ }^{[11]}$ 对 1981 年至 2001 年期间 160 多个关于灾害的实证研究进行分析, 发现自然灾 害的发生会给人们带来一些特定的心理伤害、身 心失调等短期或长期心理问题。针对自然灾害与 自杀行为之间是否具有相关性, Kôl ves ${ }^{[12]}$ 对已有 的实证研究进行了分析, 发现不同类型的自然灾 害对自杀率的影响不同, 自杀率主要受到经济条 件的影响。Hu 等 ${ }^{[13]}$ 认为灾害发生后人们在撤离和 临时安置中具有恐慌行为, 而且恐慌行为具有传 染性, 使更多受灾群体产生恐慌, Helbing ${ }^{[14]}$ 也 对突发事件下群体的恐慌逃跑非理性行为进行 了仿真模拟。突发事件下恐慌心理和非理性行为 容易导致踩踏事件, 是生命财产损失加剧的重要 影响因素 ${ }^{[15]}$ 。另外, 有些研究证明灾后人们之间 具有亲社会行为, 表现出抗逆力, Bonanno ${ }^{[16]}$ 运 用问卷调查和多变量分析发现抗逆力主要与受 灾群众的经济收入、社会支持等因素相关。van der Linden ${ }^{[17]}$, Akerlof 等 ${ }^{[18]}$ 研究者也运用实证 方法探讨了风险认知与自然灾害发生后社会结 构、经济等控制因素之间的相关性。这些研究为 探讨灾民心理行为状态的突变机理奠定了理论 和实证基础。

然而目前关于自然灾害与灾民心理行为的 研究中存在一些不足, 其一是研究者只分析了自 然灾害是否会引发人们心理及行为异常, 注重的 是自然灾害发生后人们的行为结果, 缺乏对自然 灾害导致灾民心理行为变化的过程机制研究; 其 二是现有实证方法假设控制因素与灾民心理行 为之间是线性连续的, 而这种假设在现实灾民心 理学的研究中具有局限性, Vallacher ${ }^{[19]}$ 分析了 社会心理具有动态性和复杂性, 很难用传统方法 和实证研究来描述这种现象, 需要用系统论来进 一步解释, 突变理论作为系统论的一个重要分支, 可以较好地分析内部作用机制未知的系统。

突变理论是法国数学家 Rene Thom ${ }^{[20]}$ 提出的 一种研究系统随外界控制参数连续性变化而使 系统产生跳跃、非连续性变化现象的理论。 Zeeman ${ }^{[22]}$ 对突变理论进行了进一步的研究并提 出了齐曼突变机制, 分析了尖点突变系统的几个
特征：（1）系统具有多态性，随着控制系统参数 的变化, 系统会从一个稳定状态向另一稳定状态 跳跃变化; (2) 不可达性, 系统不同状态之间不 存在绝对稳定状态, 并在现实中是不可达的; (3) 发散性, 在控制系统变化的分歧集附近两条相似 路径会使系统产生完全不同的状态; (4)滞后性, 系统经过一次扰动变化后要重新恢复到原来的 状态需要一些时间。

突变理论是以拓扑学、分叉理论、稳定性理 论为基础的理论, 它直接处理不连续性而不联系 任何特殊内在机制, 这就使它特别适合研究内部 作用未知的系统, 可以有效解释 “黑箱” 问题, 当系统具有上述突变特征时就可用突变理论来 进行解释 ${ }^{[21]}$ 。Zeeman ${ }^{[22]}$ 和 Stewart ${ }^{[23]}$ 将突变理论 应用到心理学的研究中并阐述了突变理论描述 人们态度变化的一些特定假设, van der Maas ${ }^{[24]}$ 也将突变理论应用到人们态度变化的突变机理 分析中, 并用实证数据证明了突变理论模型描述 态度变化的可行性。Scot $\mathrm{t}^{[25]}$ 针对临床心理学中 的精神分裂症、情绪失常、攻击性等其他理论无 法解释的临床心理现象用突变理论给出了有效 的解释。Flay ${ }^{[26]}$ 认为人们心理过程和社会行为不 能简单用梯度函数表示, 它满足尖点或蝴蝶突变 模型的一些特征, 并分析得到尖点突变模型更适 合描述人们行为变化。突变理论不仅能够很好地 研究社会科学问题 ${ }^{[27]}$, 也可以很好地分析企业管 理中员工的行为问题, 赵旭、胡斌等运用突变理 论分析了企业中员工的反生产行为 ${ }^{[28]}$ 、冲突行为 ${ }^{[29]}$ 、团队知识共享行为 ${ }^{[30]}$ 、员工行为突然变化 ${ }^{[31]}$ 等企业管理中的员工行为突变机理。 $\mathrm{Yiu}^{[32]}$ 分析 了建筑行业中员工冲突行为特点并建立了尖点 突变模型, 同时运用数据说明了尖点突变模型比 线性模型、回归模型更好地拟合员工冲突行为。

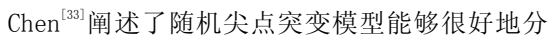
析社会行为中的理性及非理性行为, 并用实例说 明了突变模型在社会行为中的应用。

综上所述, 突变理论可以很好地分析人类的 心理态度变化, 行为突然变化等社会心理行为现 象, 为研究自然灾害发生会后灾民的心理行为状 态突变机理提供了理论依据和借鉴。但是灾民的 心理行为状态与一般突发事件和企业员工的行 为变化有很大不同, 有着自己独有的影响演变机 制, 并且目前少有文献分析灾后灾民的心理行为 状态的突变机理, 需要进一步深入分析。因此, 
Risk Analysis and Crisis Response in Big Data Era (RAC-16)

本文结合已有文献和灾区调研分析了灾民心理 行为状态的特征、影响因素, 并基于此构建了灾 民心理行为状态突变模型, 对灾民心理行为状态 突然变化问题运用突变理论进行了解释, 深入分 析了灾民心理行为的过程变化, 为及时有效实施 灾后心理干预和救援提供了理论依据。

\section{2 灾民个体心理行为状态影响因素及特征分析}

\section{1 灾民个体心理行为状态特征分析}

针对自然灾害是否会改变已有的社会经济 关系, 引发受灾群众异常行为, Casti11o $0^{[34]}$ 对洪 都拉斯遭受 1988 年米奇飓风的 30 个社区内利他 行为、信任、互惠行为等进行研究, 发现灾民行 为的均值和方差随着灾害冲击度的大小呈现非 线性变化, 适度的自然灾害冲击会提高人们之间 的合作互惠行为和亲社会行为, 但是一些家庭由 于失去住所、生存资源遭受巨大损失等经济压力, 为了维持生计而出现不合作行为, 甚至冲突行为。 时堪 ${ }^{[35]}$ 认为灾害发生后人们会有不同的心理特 征, 有时会勇敢地承担责任, 但是有时会选择退 缩、逃避。John Leach 提出的动态灾害管理模型 中也分析了灾害影响阶段人灾民的一些特殊行 为, 这些行为中有 $15 \%$ 是情绪失控和活跃行为 (overactive behavior), $75 \%$ 是紧张行为和缺 乏主动性行为, $10 \%$ 是冷静型行为和具有一定领 导力的行为 ${ }^{[36]}$ 。马德富 ${ }^{[38]}$ 对农民灾害心理和行为 特征进行分析, 得到人们心理行为具有有限理性、 短视性的特征, 短时间内往往会表现出一些情绪 行为和过激行为。朱华桂 ${ }^{[37]}$ 认为灾民个体行为反 应是人们心理的外在表现, 根据灾害发生时灾民 应对灾害的理性化程度将灾民行为分为恐慌型 行为和冷静型行为, 发现灾民的行为受心理状态 距离影响, 状态距离的微小变化会导致不同的行 为, 并进一步将灾民行为划分为 6 类, 如图 1 所 示。

通过以上分析, 可知自然灾害发生后灾民的 心理行为状态具有以下 4 个方面的特征, 如表 1 所示。

其中双模态性, 当灾害发生后, 灾民个体的 心理行为一般表现为 2 种状态, 即理性心理行为 状态和非理性心理行为状态。理性心理行为状态 下, 灾民表现出亲社会行为或利社会行为, 通常 会做出利他行为和助人行为, 这与尖点突变理论 的双模态特征较为一致; 非理性心理行为状态下, 灾民由于遭受巨大的经济损失, 失去生存资源等, 迫于生计他们可能会做出不合理的行为决策。突

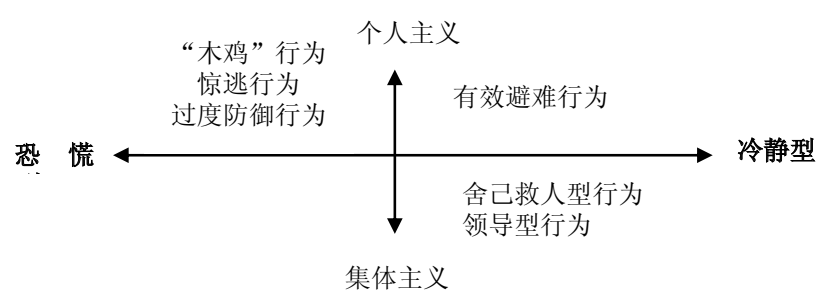

图 1. 灾民行为反应类型.

Fig.1 Victims' behavioral response types.

表 1 自然灾害情景下灾民心理行为状态特征

Table 1 Characteristics of victims' psychology and behaviorunder natural disasters scenarios

\begin{tabular}{ll}
\hline 灾民行为特征 & 具体描述 \\
\hline 1. 双模态性 & 灾害发生后人们心理行为状态只有理性和非理性两种状态, 具体表现为亲 \\
& 社会行为、互惠合作的理性行为; 冲突、不信任、恐慌的非理行为等 \\
2. 突跳性 & 灾后人们行为不是理性就是非理性, 不存在中间状态, 并且人们行为变化 \\
& 是非线性的 \\
3. 分叉性 & 人们行为状态处于临界平衡状态时, 状态距离微小变化会导致人们截然不 \\
4. 滞后性 & 同的行为 \\
\hline
\end{tabular}


Risk Analysis and Crisis Response in Big Data Era (RAC-16)

跳性是指在大多数情况下, 灾民心理行为状态不 是处于正常行为状态就是处于异常行为状态, 当 灾害发生后灾民的心理行为可能会从正常状态 跃迁到非正常状态, 心理行为跃迁并不是一个渐 变的过程, 而是一个突变过程。分叉性, 在一般 情况下当外界环境的微小变动并不会引起灾民 心理行为的巨大波动, 但灾害严重威胁到灾民的 安全后, 他们的行为状态处于临界平衡状态, 遇 到外界因素的干扰时, 这种临界平衡状态就会被 打破, 灾民个体心理行为状态会向正常行为方向 或是非正常行为方向转变, 这与突变理论的分叉 性特征相符合。滞后性, 由于自然灾害具有突发 性和不确定性等特征, 灾民可以获取的灾害信息 极少, 这种情况下, 个体在进行决策时, 就需要 收集整理信息, 并且需要考虑个人安全、损失等 因素, 无法及时作出决策, 具有明显的滞后性。

由此可见, 自然灾害情景下灾民心理行为状 态的特征符合Zeeman 所阐述的齐曼突变的特点, 可以用尖点突变模型来刻画灾民的心理行为状 态的突变机理。

\section{2 灾民个体心理行为影响因素分析}

突发事件下人们心理行为受许多因素影响, 孙多勇 ${ }^{[39]}$ 将个体恐惧行为影响因素分为事件本 身、社会、个体及对个体与灾害事件相关因素四 类, 并建立了个体恐惧模型, 分析了个体的恐惧 主要来源于心理预期。马庆国等 ${ }^{[40]}$ 认为突发事件 下人的行为是心理、周围氛围和信息的函数, 并 分析了各类因素对人的行为的影响。也有学者认 为风险认知是决定灾后人们心理行为状态的关 键因素, 风险认知的高低决定了灾民不同的心理 行为状态 ${ }^{[41]}$, 人们的风险认知是由不同因素决定
的, 如对社会的信任度、个体灾害经历、心理距 离等变量的影响 ${ }^{[22]}$ 。

但是行为科学认为人们的各种行为都是有 一定的动机引起的, 而这种动机又产生于人们身 体的各种需要 ${ }^{[45]}$ 。人们为了满足某种需要就要确 定自己的行为目标, 进而采取一系列的行为。董 惠娟等 ${ }^{[46]}$ 通过 2000 多份问卷对唐山地震后人们 的心理相关问题进行了研究, 发现灾后人们物质 性需求异常激烈, 这是因为自然灾害对人们的生 存条件产生的破坏力比其他突发事件更大, 求生 本能促使人们更关注物质性需求。同时也发现灾 后一段时间人们仍有被安慰和被关注的心理需 要。2013 年 7 月至 8 月期间课题组奔赴雅安地震 重灾区芦山县进行实地调研, 调研时间是雅安地 震发生后 3 个月, 灾民情绪基本稳定, 处于灾后 恢复期, 通过访谈和个体问卷得到灾后人们心理 痛苦的压力感主要来源于经济损失, 政府虽然给 予了一定程度的补贴, 但是还远远不能弥补地震 带来的损失, 虽然灾后人们情绪慢慢恢复正常, 但是通过创伤后应激障碍自评表我们发现仍有 $80 \%$ 以上的灾民有焦虑、恐慌等情绪, 不能很好 地投入灾后重建工作中, 他们很渴望人与人之间 的情感交流, 具有一定的亲社会行为。对调研资 料总结得到灾后恢复期人们的需求主要是两个 方面: 物质需求和情感需求。通过物质需求来满 足灾后的衣食住行及生命安全, 通过情感需求满 足自己的归宿感、消除灾后的孤独感和抑郁。这 两种需求的满足程度决定了受灾群众的风险认 知水平, 进而决定了恐慌程度及行为的理性程度, 风险认知在需求和心理行为之间起中介作用。通 过以上文献分析和实地调研总结得到自然灾害 情景下灾民的心理行为影响因素如表 2 所示。

表 2. 自然灾害情景下灾民心理行为影响因素分析表

Table 2. Factors of victims' psychology and behavior under natural disaster scenario

\begin{tabular}{|c|c|c|}
\hline 影响因素 & 具体描述 & 资料来源 \\
\hline 物质需求因素 & $\begin{array}{l}\text { 满足受灾群众衣食住行及医疗卫生等 } \\
\text { 方面的基本物资, 主要维持灾民基本生 } \\
\text { 活和生命安全 }\end{array}$ & 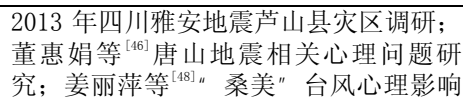 \\
\hline 情感需求因素 & $\begin{array}{l}\text { 来自心理医疗卫生人员、志愿者、亲朋 } \\
\text { 好友的安慰与心理辅导, 一种心理抚㓪 } \\
\text { 需求 }\end{array}$ & $\begin{array}{l}\text { 因素研究; 孙燕娜等 }{ }^{[49]} \text { 救灾需求内涵模 } \\
\text { 式研究; RR Dynes, et } \mathrm{al}^{[51]}\end{array}$ \\
\hline 风险认知 & $\begin{array}{l}\text { 对有关风险的信号进行加工、过滤, 在 } \\
\text { 主观上对风险产生认知, 并做出判断和 } \\
\text { 评价, 形成关于风险的自我理解, 对推 } \\
\text { 动人们采取何种风险行为有重要作用 }\end{array}$ & $\begin{array}{l}\text { Sheeran P, et al, }{ }^{[41]} \text {; } \\
\text { Milfont }{ }^{[42]} \text {; Spence , et al }{ }^{[43]} \text {; Wachinger } \\
\mathrm{Gl}^{[43]} \text {; } \\
\text { Naylor } \mathrm{R}^{[52]} \text {;Becker } \mathrm{G} \text {, et } \mathrm{al}^{[53]}\end{array}$ \\
\hline
\end{tabular}




\section{Risk Analysis and Crisis Response in Big Data Era (RAC-16)}

\section{3 灾民个体心理行为变化的形成机制分析}

本文研究的灾民个体心理行为界定为自然 灾害发生后恢复期阶段灾民的心理行为, 通过以 上文献分析和实地调研, 可知这一时期物质需求 因素和情感需求因素会影响人们的心理认知评 价, 进而决定人们的行为, 灾民的心理行为可能 会在恐慌型行为和冷静型行为之间发生突然变 化, 这种突然变化是由人们心理状态距离感知的 突变引起的。自然灾害的发生打破人们已有的心 理平衡状态, 会产生一种状态距离 ${ }^{[37]}$, 这种状态 距离超过一定阈值时人们的心理行为就会处于 不同的状态, 产生一种跳跃式变化, 本文在朱华 桂 $^{[37]}$ 提出的灾民心理行为框架的基础上提出自 然灾害下灾民心理行为的变化机制模型, 如图 2 所示。自然灾害的风险认知, 是灾民在灾害发生 时对灾害风险信息进行加工、判断与评价，风险
认识越大, 越容易引起灾民人体心理上的恐慌, 越容易出现非理性的恐慌行为。风险认知是引起 灾民恐慌行为的关键中间变量, 影响灾民心理和 行为的因素 (如物质需求因素和情感需求因素) 都是通过心理风险认知评价对灾民心理和行为 发生作用。

由形成机制模型可知, 自然灾害发生后灾民 心理行为状态有恐慌型行为和冷静型行为两种 相对稳态。恐慌型行为是一种负的稳态, 在这种 状态下灾民会有恐惧、与其他灾民争吵、冲突行 为, 甚至会哄抢物资等威胁社会稳定与秩序的风 险行为。但是当灾民的心理状态距离逐渐缩小到 心理感知阈值时人们的行为可能会突然转化为 冷静型行为, 如积极地投入灾后救援及重建工作 中，帮助其他灾民等亲社会行为。

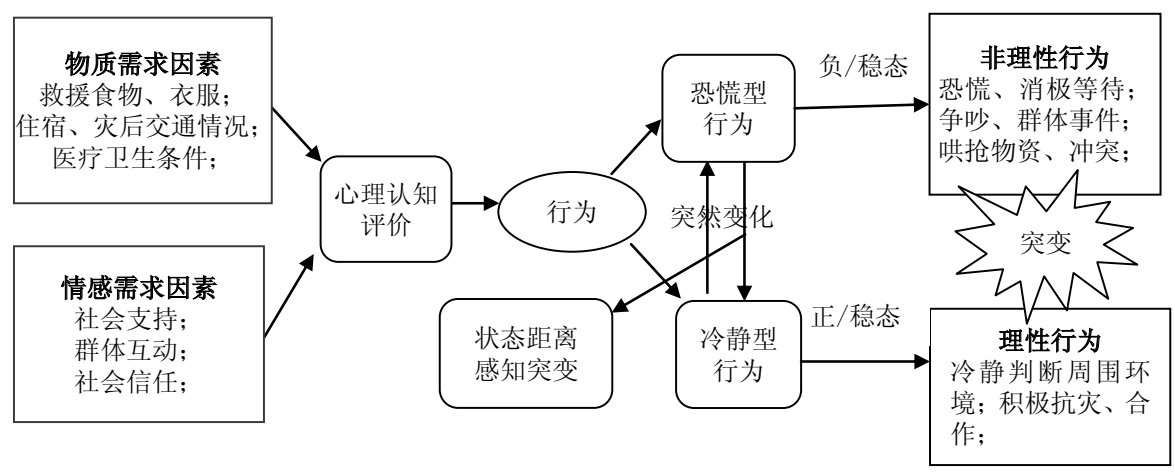

图 2. 自然灾害恢复期灾民个体心理行为变化形成机制图.

Fig. 2 Formation mechanism for individual victim's psychological behavior changes during the recovery stage of natural disasters.

\section{3 灾民个体心理行为尖点突变模型建立与突变 机理分析}

\section{1 灾民个体心理行为尖点突变模型构建}

根据灾民个体心理行为状态形成机制，可将 灾民的个体心理行为状态看成是由灾后物质需 求因素和情感需求因素耦合共同决定的。由马斯 洛需求理论可知, 人们的需求可以分为五大类, 其中生理需求放在首位, 生理需求包括水、食物 等的需求。灾害发生后, 灾民对物质的需求远大 于情感需求, 物质需求因素的重要程度要高于情 感需求因素。当物质需求满足后, 灾民的情感需
求也得到了满足。将灾民的个体心理行为 $x$ 看作 状态变量, 将物质需求因素满足程度 $u$ 和情感需 求因素满足程度 $v$ 看作控制灾民个体心理行为的 控制变量, 个体心理行为 $x$ 用心理状态感知度测 量, $u, v, x \in[-1,1]$, 用 $V$ 表示灾民个体心理状态 行为尖点突变模型的势函数 ${ }^{[20]}$ 。突变理论中在处 理控制变量时, 重要程度相对大的控制变量放在 前面, 相对次要的放在后面, 因此势函数如公式 (1) 所示

$$
V(x, u, v)=x^{4}+u x^{2}+v x
$$

灾民心理行为曲面 $M$ 是突变模型均衡点的 集合, 即为突变模型的平衡曲面, 如公式 (2) 
Risk Analysis and Crisis Response in Big Data Era (RAC-16)

所示

$$
M=\frac{\partial V(x, u, v)}{\partial x}=4 x^{3}+2 u x+v=0
$$

奇点集合 $N$ 为势函数 $\mathrm{V}$ 的二阶导数, 如公式 (3) 所示

$$
N=12 x^{2}+2 u=0
$$

灾民心理行为突变区域 $B$ 为突变模型的分歧点集, 是 (2) 式与 (3) 式联立消去 $x$ 得到的解, 如公 式 (4) 所示

$$
B=8 u^{3}+27 v^{2}=0
$$

公式 (1) 为灾民个体心理行为状态平衡曲 面方程, 假设灾民物质和情感需求满足程度、心 理状态感知度为区间 $[-1,1]$ 之间的数值, 物质和 情感需求满足程度越大心理状态感知度越小。根 据公式(1)用MATLAB 模拟控制变量在区间 $[-1,1]$ 之间连续变化对状态变量的影响, 模拟数据是 1000 个, 可得到灾民个体心理状态尖点突变模型 的平衡曲面如图 3 所示, 然后根据此方法运用公 式 (4) 得到灾民个体心理行为尖点突变的分歧 集, 即图 3 中的褶皱面在控制面上的投影, 如图

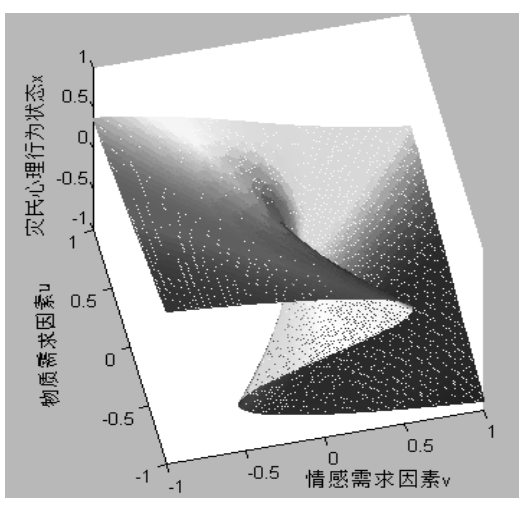

图 3. 灾民个体心理行为状态尖点突变平衡曲面.

Fig. 3 The cusp catastrophe model equilibrium surface of individual victim's psychological behavior.

图 5 中灾民个体心理行为变化曲线为 $a \rightarrow b$ 或 $d \rightarrow e$ 时, 灾民的心理状态感知距离 $\Delta x$ 逐 渐变大, 但是还没超过心理感知阈值, 灾民的冷 静程度逐渐变小, 变得越来越不理性, 仍是一种 渐变, 还没发生突变, 当分别经过 $b$ 点、 $\mathrm{e}$ 点时, 灾民心理行为曲线经过分歧区域 B, 从冷静型行 为瞬间突变为恐慌型行为, 但是其投影到控制平
4 所示。在这 1000 个数据中当物质需求满足程度 $u$ 和情感需求因素满足程度 $v$ 满足公式 (4) 的分 歧方程时, 灾民个体心理行为状态曲线的投影在 控制面上会经过分歧集, 导致灾民个体心理行为 状态发生突变。

\section{2 灾民个体心理行为突变机理分析 \\ 根据灾民个体心理行为尖点突变平衡曲面} 图 3 和灾民个体心理行为状态分歧集图 4, 对其 进行旋转和简化, 用Visio 画图软件绘制出图 3 和图 4 的直观图形, 图 4 为图 3 中的褶皱曲面在 控制面的投影, 得到自然灾害发生后灾民的个体 心理行为状态演化直观模型, 如图 5 所示, 据此 可以分析灾民个体心理行为发生的原因、过程及 程度。图 5 中的平衡曲面分为上叶、中叶、下叶 三部分, 其中上叶代表的是灾民冷静型行为, 下 叶代表的是灾民恐慌型行为, 灾民个体心理行为 在平衡曲面的上、下叶时是处于相对稳定的状态, 中叶是一个不稳定状态, 即灾民个体心理行为发 生突变的区域。

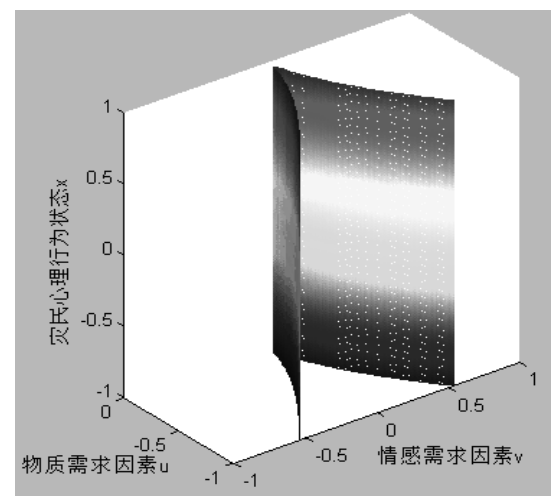

图 4. 灾民个体心理行为状态尖点突变分歧集.

Fig. 4 The bifurcation set of cusp catastrophe model of individual victim's psychological behavior.

面分歧点集的曲线 $\mathrm{f} 1$ 与 $\mathrm{f} 2$ 之间是有差异的, 说明 这两种不同的行为变化导致的灾民的恐慌程度 是不同的。当灾民心理行为状态曲线为 $\mathrm{b} \rightarrow \mathrm{c}$ 时, $\Delta \mathrm{f} 1=x\left(u_{b}, v_{b}\right)-x\left(u_{c}, v_{c}\right)$, 而当灾民心理行为 状态曲线为 $\mathrm{e} \rightarrow \mathrm{f}$ 时, $\Delta \mathrm{f} 2=x\left(u_{e}, v_{e}\right)-x\left(u_{f}, v_{f}\right)$, 通过控制面的分歧集很容易发现 $\Delta \mathrm{f} 2>\Delta f 1$, 这 说明心理行为曲线 $\mathrm{d} \rightarrow \mathrm{e} \rightarrow \mathrm{f}$ 产生的危害程度更 


\section{Risk Analysis and Crisis Response in Big Data Era (RAC-16)}

大，这时灾民心理行为可能是哄抢物资、与他人 冲突甚至是群体性事件，而心理行为曲线为 $a \rightarrow b \rightarrow c$ 时，灾民可能只是个体的恐慌、消极 等待救援等行为, 对社会稳定与秩序构不成很大 威胁。

通过以上模型分析，可知当灾民的物质需 求满足程度和情感需求满足程度不断变小时, 灾 民的心理行为状态会从冷静型行为跳跃为恐慌 型行为, 对社会的稳定与秩序构成巨大威胁, 但 是物质需求因素和情感需求因素对灾民的心理 行为变化的作用程度不同。分两种情形进行讨论:

（1）情形一: 当灾民个体物质需求满足程 度大于零时, 即 $u>0$ 时, $8 u^{3}+27 v^{2}>0$, 公 式 (4) 无解, 灾民个体心理行为曲线不经过分 歧集, 随着 $v$ 的连续变化, 灾民的心理行为状态 只是连续性变化, 不会产生跳跃现象;

（2）情形二: 当灾民个体物质需求满足程 度不大于零时, 即当 $u \leq 0$ 时, $8 u^{3}+27 v^{2} \leq 0$, 公式 (4) 有解, 由图 4 可知 $v$ 从 -1 逐渐变为 +1
时，灾民心理行为曲线经过了分歧集，灾民的心 理行为状态发生突然跳跃现象。

由突变理论可知, 物质需求满足程度 $u$ 为分 裂因子, 即 $u$ 的变化会使平衡曲面 $M$ 分裂, 产生 不同的状态，决定了灾民心理行为是否会发现突 变。如图 5, 当物质需求满足程度达到较高水平 时, 即 $u$ 接近 1 时, 随着情感需求满足程度的连 续性变化, 灾民个体心理行为曲线 $\mathrm{CP}$ 从平衡面 的上叶连续变化到下叶, 其投影在控制面上没有 经过分歧集, 表明灾民的冷静程度逐渐较低, 但 不会突然演变为威胁社会稳定的非理性行为, 并 且这一变化过程是需要一段时间的; 而情感需求 因素 $v$ 为正则因子, 即 $u>0$ 时 $v$ 的变化只引起状 态变量的光滑变化。通过图 4 和图 5 可以得到物 质需求满足程度 $u$ 不断减小到 0 时, 情感需求因 素 $v$ 微小变化会产生截然不同的行为路径 $n 1$ 和 $n 2$ 。 $n 1$ 曲线经过分歧区域达到平衡曲面的下叶, 产生恐慌型行为; 而 $n 2$ 曲线经过分歧达到平衡曲 面的上叶, 产生冷静型行为。

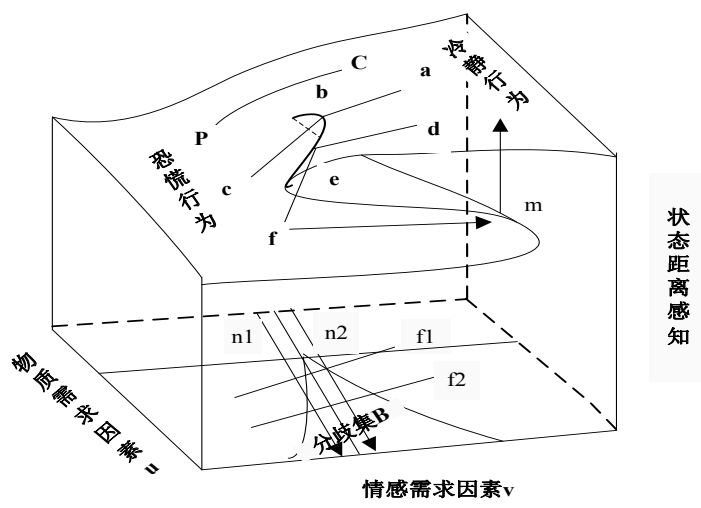

图 5. 自然灾害恢复期灾民个体心理行为状态演化直观模型.

Fig. 5 The intuitive evolution model of individual victim's psychological behavior of nature disaster during recovery stage.

\section{4 灾民个体心理行为尖点突变模型的局部验证}

自然灾害情景下灾民个体心理行为突变是 瞬间的, 并且是动态变化的, 其突变过程很难被 捕捉到, 很难用实际数据描述灾民个体心理行为 的尖点突变过程, 本文采用问卷调研的方法对物 质需求和情感需求两种控制变量类型进行局部 验证，并阐明模型的有效性。

本研究对芦山县灾民进行随机抽样调研, 共
发放问卷 405 份, 回收有效问卷 306 份, 问卷有 效率为 $75.5 \%$ 。采用创伤后应激障碍检查表一普通 版 (PCL-C) 测量灾民的心理行为, 因为 PTSD (Post-traumatic stress disorder) 既能反应 灾民的心理状态（如烦躁、抑郁）又能体现灾民 的行为 ${ }^{[54]}$ 。用社会支持、社会信任和社会网络来 测量灾民情感需求与 PTSD 之间的关系, 该部分 问卷采用 Liket 5 点量表, 数字越大表示获得的 
情感支持越多。经济收入越低的家庭越贫穷, 社 会脆弱性越大, 经济承受力越差, 应对灾害能力 越低, 灾后物质需求程度更大 (Cutter, 2003) ) $^{[55]}$, 因此可以用经济收入水平测量物质需求与 PTSD 之间的关系。根据当地灾民月均收入水平划分为 5 个层次, $1=1000$ 元及以下, $2=1001-2000$ 元, $3=2001-3000$ 元, 4=3001-4000 元, 5=4000 元以 上, PTSD 自评表总分越高 PTSD 症状越明显。为

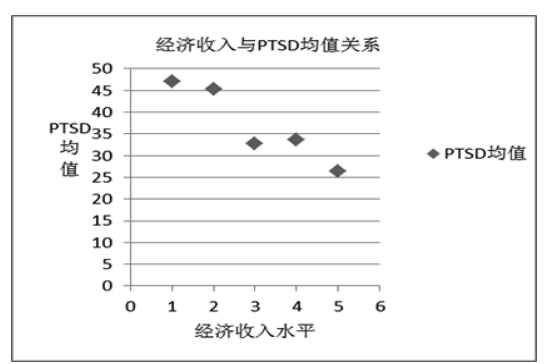

图 6. 经济收入水平与 PTSD 均值散点图.

Fig.6 Scatterplot of income level and the average of PTSD.

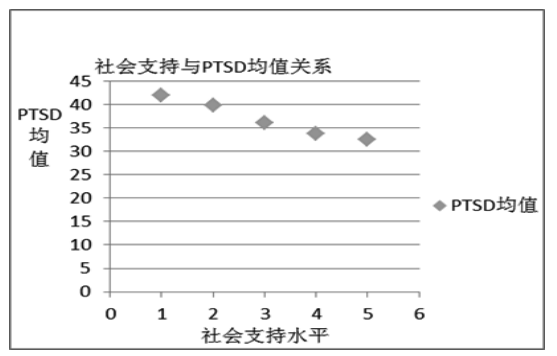

图 8 社会支持与 PTSD 均值散点图.

Fig. 8 Scatterplot of social support level and the average of PTSD.

由图 6 可知, 随着月均收入水平的增大 PTSD 均值逐渐减小, 但当月均收入达到 2000 元时, PTSD 均值突然减小, 使得 PTSD 均值的变化呈现 非线性关系。由图 7、图 8 和图 9 可知, 社会网 络、社会支持和社会信任等情感支持水平逐渐提 高 PTSD 均值呈现逐渐变小趋势, 但是情感支持 水平与 PTSD 均值的变化呈现线性关系, 不会产 生突然跳跃现象。这可以说明将物质需求满足程 度作为分裂因子, 情感需求满足程度作为正则因 子是基本符合实际情况的。这与马斯洛的需求层 次理论也基本相符, 人们的需要是分层次的, 只
了描述经济收入、情感支持与 PTSD 之间的关系, 用 SPSS19. 0 分别对 306 位灾民按经济收入水平 和情感支持度划分为 5 组, 并分别得到各组的 PTSD 均值, 然后画出经济收入水平与 PTSD 均值 的散点图, 如图 6 所示; 社会网络与 PTSD 均值 的散点图, 如图 7 所示; 社会支持与 PTSD 均值 的散点图, 如图 8 所示; 社会信任水平与 PTSD 均值散点图, 如图 9 所示。

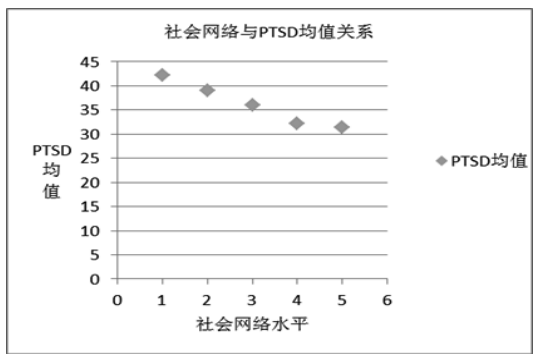

图 7. 社会网络与 PTSD 均值散点图.

Fig.7 Scatterplot of social network level and the average of PTSD.

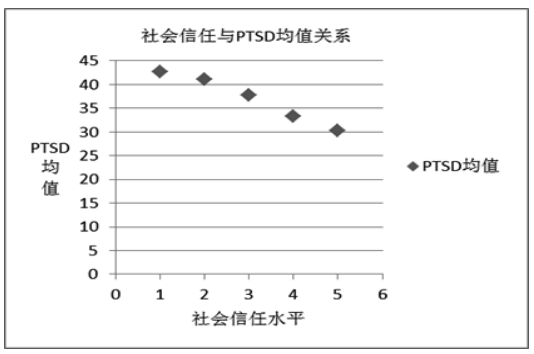

图 9 社会信任水平与 PTSD 均值散点图.

Fig.9 Scatterplot of social trust level and the average of PTSD.

有满足了底层的需要才会关注更高层次的需要。 自然灾害发生后, 人们面临最大的威胁就是生存 威胁, 人们最关注的需要就是生理需要和安全需 要。同时, 人又具有社会属性, 需要人与人之间 的交流来满足情感需求, 尤其是灾后心理创伤期。

\section{5 灾民个体心理行为应对策略}

自然灾害发生 2 至 3 个月后, 灾区开始慢慢 恢复, 受灾群众情绪相对比较稳定, 有时会非常 理性地积极投入灾后重建工作中, 但是有时会突 然变得不够理性, 与他人产生一些冲突, 甚至是 
Risk Analysis and Crisis Response in Big Data Era (RAC-16)

群体性事件, 这除了与个人特质有关, 更受人们 当时需求因素的影响。灾民的心理创伤在需求因 素的作用下以外在行为的方式表现出来。通过以 确保灾民第一时间领取救援物资。通过以上分析 可知物质需求满足程度决定了灾民心理行为是 否会发生突变, 当物质需求满足程度 $u>0$ 时, 灾民心理行为只会发生连续性变化, 不会经过分 歧集突变为恐慌型行为, 但当 $u$ 逐渐到 0 时, 灾 民心理行为曲线就会经过分歧集, 心理状态行为 会产生跳跃。所以, 灾后要第一时间通过多种途 径满足灾民的物质需求, 尤其是对于低收入人群, 减轻其关于物质方面的相对剥夺感, 提高灾害应 对能力和经济承受力。

（2）建立持久的心理干预机制，提供健全 的情感支持体系。灾后恢复期人们除了关心自己 的衣食住行之外, 有被关心、被关注的情感需求。 自然灾害给灾民带来巨大的经济损失和精神创 伤, 往往缺乏重建的信心和勇气, 这时就需要政 府和社会各界人士提供一定的情感支持, 帮助灾 民恢复重建的信心，通过上述分析也可以看到当 灾民的物质需求满足程度逐渐减低到 0 时, 情感 需求的微小变化会引起灾民完全不同的行为反 应。因此, 灾后恢复期也要高度重视灾民的情感 需求, 充分利用各种资源帮助灾民恢复社会人际 关系网络。

\section{6 结论}

自然灾害恢复期灾民有时会表现出较高的 理性程度, 积极投入到灾后重建工作中, 但有时 会从冷静型行为突然变化为恐慌型行为, 甚至产 生冲突行为。针对这一问题, 本文根据文献资料 和灾区调研分析了灾民个体心理行为状态影响 因素, 运用突变理论构建了灾民个体心理行为突 变模型, 刻画了灾民个体心理行为状态演化的直 观模型, 探讨了灾民个体行为突变机理, 并用实 际调研数据对提出的模型进行了局部的验证。通 过分析得到物质需求满足程度是灾民心理行为 突变的决定性因子, 情感需求满足程度是灾民心 理行为突变的非决定性因子, 但当物质需求满足 程度较低到零时, 情感需求的微小变化会使灾民 产生截然不同的心理行为。因此, 灾后要建立完 善的救援物资分配体系，第一时间满足灾民的物 质性需求, 同时也不能忽略灾民的情感需求, 需 要政府部门建立持久的心理干预机制, 为灾民提 供健全的情感支持, 防止灾民微小的情感变化引
上分析, 可知这一时期要充分满足灾民的各种需 求, 但是满足的优先顺序和程度不同。

（1）建立完善的救援物资分配运送体系, 起的非理性行为。本文虽然分析了灾民个体心理 行为的突变机制, 但模型的验证具有局限性, 需 要考虑个人特质 (如年龄、性别、文化程度等) 、 信息传播、政府心理干预、人口密度、社会结构、 灾害类型等多种因素的相互作用对灾民心理行 为的演化影响。同时, 本文调研数据有限, 未来 仍需要大量的实证资料来进一步验证。

\section{参考文献}

[1] 民政部. 2014 年全国自然灾害基本情况

[2] Cameron L, Shah M. Risk-taking behavior in the wake of natural disasters. National Bureau of Economic Research, 2013.

[3] Kunimatsu M M, Marsee M A. Examining the presence of anxiety in aggressive individuals: The illuminating role of fight-or-flight mechanisms //Child \& Youth Care Forum. Springer US, 2012, 41(3): 247-258.

[4] 刘正奎, 吴坎坎, 王力. 我国灾害心理与行为研 究. 心理科学进展, 2011, 19(8): 1091-1098.

[5] Hu Z H, Sheu J B. Post-disaster debris reverse logistics management under psychological cost minimization. Transportation Research Part B: Methodological, 2013, 55: 118-141.

[6] Rennemo S J, Rø K F, Hvattum L M, et al. A three-stage stochastic facility routing model for disaster response planning. Transportation Research Part E: Logistics and Transportation Review, 2014, 62: 116-135.

[7] Xuanhua Xu, Chenguang Cai, et al. Research on Society Risk Evolution Mechanism and Counter Measures in Severe Emergency Infectious Disease - in the Case of H7N9 Avian Influenza. Journal of Risk Analysis and Crisis Response, 2015, 5(1):54-65.

[8] Chae J, Thom D, Jang Y, et al. Public behavior response analysis in disaster events utilizing visual analytics of microblog data. Computers \& Graphics, 2014, 38: 51-60.

[9] 范维澄. 国家突发公共事件应急管理中科学问 题的思考和建议. 中国科学基金, 2007, 21(2): 71-76.

[10] Doherty T J, Clayton S. The psychological impacts of global climate change. American Psychologist, 2011, 66(4): 265.

[11] Norris F H, Friedman M J, Watson P J, et al 60,000 disaster victims speak: Part I. An empirical review of the empirical literature, 1981-2001. Psychiatry: Interpersonal and biological processes, 2002, 65(3): 207-239.

[12] Kõlves K, Kõlves K E, De Leo D. Natural disasters and suicidal behaviours: A systematic literature review. Journal of Affective Disorders, 2013,146(1):1-14.

[13] Hu Z, Sheu J, Xiao L. Post-disaster evacuation and temporary resettlement considering panic and panic spread. Transportation Research Part B: 
Methodological, 2014,69:112-132.

[14] Helbing D, Farkas I, Vicsek T. Simulating dynamical features of escape panic. Nature, 2000, 407(6803): 487-490.

[15] 朱华桂. 突发事件中个体行为的科学决策. 科学 对社会的影响, 2008(04):31-37.

[16] Bonanno G A, Galea S, Bucciarelli A, et al. What predicts psychological resilience after disaster? The role of demographics, resources, and life stress. Journal of consulting and clinical psychology, 2007, 75(5): 671 .

[17] van der Linden $S$. The social-psychological determinants of climate change risk perceptions: Towards a comprehensive model. Journal of Environmental Psychology, 2015, 41:112-124.

[18] Akerlof K, Maibach E W, Fitzgerald D, et al. Do people "personally experience" global warming, and if so how, and does it matter? Global Environmental Change, 2013, 23(1): 81-91.

[19] Vallacher R R, Nowak A. The emergence of dynamical social psychology. Psychological Inquiry, 1997, 8(2): 73-99.

[20] Thom R. Stabilite structurelle et Morphogenese. New York: Benjamin

[21] 桑博德 [英]. 凌复华译. 突变理论入门.上海: 上海 科学技术文献出版社, 1983 .

[22] Zeeman E C. Catastrophe theory. Springer Berlin Heidelberg, 1979.

[23] Stewart I N, Peregoy P L. Catastrophe theory modeling in psychology. Psychological bulletin, 1983, 94(2): 336.

[24] Van der Maas H L J, Kolstein R, Van Der Pligt J. Sudden transitions in attitudes. Sociological Methods \& Research, 2003, 32(2): 125-152.

[25] Scott D W. Catastrophe theory applications in clinical psychology: A review. Current Psychology, 1985, 4(1): 69-86.

[26] Flay B R. Catastrophe theory in social psychology: Some applications to attitudes and social behavior. Behavioral Science, 1978, 23(4): 335-350.

[27] 姜璐, 于连宇. 初等突变理论在社会科学中的应 用. 系统工程理论与实践, 2002, 10(130): 3-5.

[28] 赵旭, 胡斌. 基于突变理论的企业员工反生产行 为研究. 管理科学, 2012, 25(4): 44-55.

[29] 赵旭, 胡斌. 集成突变论和演化博亦的企业员工 冲突行为研究. 运筹与管理, 2014, 23(4): 228-237.

[30] 徐岩, 胡斌, 杨永清. 团队知识共享行为的随机 突变模型及仿真. 运筹与管理，2013，22(5): 240-249.

[31] Hu B, Xia N. Cusp catastrophe model for sudden changes in a person's behavior. Information Sciences, 2015, 294: 489-512.

[32] Yiu K T W, Cheung S O. A catastrophe model of construction conflict behavior. Building and environment, 2006, 41(4): 438-447.

[33] Chen D. Stochastic Cusp Catastrophe Model and its Sample Size Determination in Social Behavior Research//142nd APHA Annual Meeting and Exposition (November 15-November 19, 2014). APHA, 2014

[34] Castillo M, Carter M. Behavioral responses to natural disasters. Unpublished Manuscript, 2011.

[35] 时堪.灾难心理学.北京:科学出版社,2010:16-50.

[36] Vorst H C M. Evacuation models and disaster psychology. Procedia Engineering, 2010,3:15-21

[37] 朱华桂. 突发灾害情境下灾民恐慌行为及影响 因素分析. 学海, 2012 (5): 90-96.

[38] 马德富. 论农民灾害心理及行为选择的有限理 性及对策. 湖北社会科学, 2010 (3): 76-78

[39] 孙多勇. 突发性社会公共危机事件下个体与群 体行为决策研究.长沙: 国防科技大学, 2005 .

[40] 马庆国, 王小毅. 非常规突发事件中影响当事人 状态的要素分析与数理描述. 管理工程学报, 2009, 23(3): 126-130.

[41] Sheeran P, Harris P R, Epton T. Does heightening risk appraisals change people's intentions and behavior? A meta-analysis of experimental studies. Psychological bulletin, 2014, 140(2): 511.

[42] Milfont T L. The interplay between knowledge, perceived efficacy, and concern about global warming and climate change: a one - year longitudinal study. Risk Analysis, 2012, 32(6): 1003-1020.

[43] Spence A, Poortinga W, Pidgeon N. The psychological distance of climate change. Risk Analysis, 2012, 32(6): 957-972.

[44] Yanran Yang, Lina Jin, etal.Crisis Communication about Nuclear Accidents with Psychological Approaches. Journal of Risk Analysis and Crisis Response, 2015, 5(3): 169-177.

[45] 周三多,陈传明,鲁明泓.管理学-原理与方法.上海: 复旦大学出版社,2009,69-80.

[46] 董惠娟, 李小军, 杜满庆, 等. 地震灾害心理伤 害的相关问题研究. 自然灾害学报, 2007, 16(1): 153-158.

[47] Psychological traumas.Journal of Natural Disasters, 2007, 16(1): 153-158.(in Chinese)

[48] 姜丽萍, 姚岗, 王玉玲, 等. 桑美台风灾害对幸 存者心理影响的定性研究. 中国心理卫生杂志, 2008(12):929-933.

[49] 孙燕娜, 王玉海, 廖建辉. 救灾需求内涵模式及 其指标体系与救助评估研究. 经济与管理研究, 2010 (6): 85-94.

[50] Index System and Aid Assessment.Research on Economic and Management, 2010 (6): 85-94.(in Chinese)

[51] Dynes R R, Quarantelli E L, Kreps G A. A perspective on disaster planning. Ohio State Univ Columbus Disaster Research Center, 1972.

[52] Naylor R, Courtney P. Exploring the social context of risk perception and behaviour: Farmers' response to bovine tuberculosis. Geoforum, 2014, 57: 48-56.

[53] Becker G, Aerts J, Huitema D. Influence of flood risk perception and other factors on risk - reducing behaviour: a survey of municipalities along the Rhine. Journal of Flood Risk Management, 2014, 7(1): 16-30.

[54] 向莹君, 熊国玉，董毅强，等. 汶川地震灾区 1960 名中学生创伤后应激障碍症状调查. 中国 心理卫生杂志, 2010 (1): 17-20.

[55] Cutter S L, Boruff B J, Shirley W L. Social vulnerability to environmental hazards. Social science quarterly, 2003, 84(2): 242-261. 